\title{
Workforce Diversity Management: Biggest Challenge Or Opportunity For 21st Century Organizations
}

\author{
Main Authors'. Preeti bedi; Co- Authors'. Poonam lakra; Co- Authors'. Ekanshi \\ Gupta; \\ Assistant professor BhaginiNivedita College, University of Delhi \\ Assistant professor BhaginiNivedita College, University of Delhi \\ Assistant professor Swami Shraddhanand College, University of Delhi
}

\begin{abstract}
Management being a social discipline deals with the behavior of people and human insight. Hence, workforce diversity is the biggest challenge and at the same times the biggest opportunity for the 21st century managers. "Diversity Management" is a strategy to promote the perception, acknowledgement and implementation of diversity in organizations. Workforce diversity has significant implications for the management. The managers will be required to shift their approach from treating each group of workers alike to recognizing differences among them and following such policies so as to encourage creativity, improve productivity, reduce labor turnover and avoid any sort of discrimination. Successful diversity management policy and program will eventually make a huge difference in the communication among employees and the general productivity of the organization. Today's organization need to recognize and manage workforce diversity effectively. The study is conducted to explore how companies manage workforce diversity and its consequences to the company's existence as well as examine how companies' deal with challenges that comes with employees from diverse cultural backgrounds. Is workforce diversity related to organization performance and productivity and its effect on human resource management? The research therefore answers the question 'Has workplace diversity contributed to organizational success`.

Keywords: diversity, management, productivity, work force, contribution
\end{abstract}

\section{Introduction}

Workplace diversity is the issue of people, focus on the differences and similarities that people bring to an organization. We are live in an increasingly multicultural society. It is a melting pot or a stew. Successful organizations recognize the need for immediate action and are ready and willing to spend resources on managing diversity in the work place. Similar to globalization, diversity and social issues have had a dramatic effect on the study and application of management and organizational behavior. In the past, diversity was treated as a legal issue; that is for well over 45 years it has been directly against the law to discriminate against any one, on any basis. Now organizations are becoming to realize that diversity is not just something to deal with, but instead a reality to build onto make a stronger more competitive enterprise.

\section{Meaning Of Work Force Diversity}

Workforce diversity is simply how diverse is the workforce. Are there several people with a mix of cultures, age, background, race, and etc? If there are many people that have differences. According to Moorhead and Griffin "Workforce diversity is basically concerned with the similarities and differences in such characteristics as age, gender, ethnic, heritage, physical abilities and disabilities, race and sexual orientation among the employees of the organization" As we enter the 21st century, workforce diversity has become an essential business concern. In the so-called information age, the greatest assets of most companies are now on two feet (or a set of wheels). Undeniably, there is a talent war raging. No company can afford to unnecessarily restrict its ability to attract and retain the very best employees available.

Generally speaking, the term "Workforce Diversity" refers to policies and practices that seek to include people within a workforce who are considered to be, in some way, different from those in the prevailing constituency. In this context, here is a quick overview of seven predominant factors that motivate companies, large and small, to diversify their workforces.

\section{Benefits Of Workforce Diversity}

Diversity has multiple benefits to the workplace. One of the major principles of diversity says that a company that has diverse employees has a greater understanding of the global marketplace. According to DiversityWorking.com, employers reported that their diverse organizations benefit from a variety of viewpoints, higher productivity and profit due to company cultures that encourage employees to perform to their highest 
ability. Employers may also recognize immediate benefits of workplace diversity. Customers who speak different languages or come from overseas may require customer service in their language. In industries such as marketing and advertising, knowing what consumers across different backgrounds want is crucial to success. Advantages of having diverse workforce:

1) High level of Productivity: When management takes the welfare of its workers at heart by means of offering them proper compensation, health care and employee appraisal, It enables workers to feelsthey belong to the company irrespective of their cultural background by remaining loyal and hardworking which helps to increase the company's productivity and profit.

2) Exchange of varieties of ideas and Team work: A single person taking on multiple tasks cannot perform at the same pace as a team could; therefore each team member brings to the table different ideas and offers a unique perspective during problem solving to effectivelyarrive at the best solution at the shortest possible time.

3) Learning and growth: Diversity at the workplace creates an opportunity for employee's personal growth When workers are being exposed to new cultures, ideas and perspectives, it can help each person to intellectually reach out and have a clearer insight of their place in the global environment and hence their own surroundings

4) Effective Communication: Workplace diversity can immensely strengthen a company's relationship with some specific group of customers by making communication more effective. A customer service personnel or representatives can be paired up with customersfrom their specific area or location, making the customer feel at home with the representative and thus with the company.

5) Diverse Experience: Employee and their co-workers that come from a diverse background bring to the table some amount of unique perceptions and experience during teamwork or group tasks. Pooling the diverse skills and knowledge of culturally distinct employees together can immensely benefit the company by strengthening the responsiveness and productivity of the team to adapt to the changing conditions.

\section{Managing Diversity Is An Important And Difficult Task}

To address diversity issues certain question to be answered:

- what policies, practices, and ways of thinking and within our organizational culture have differential impact on different groups?

- What organizational changes should be made to meet the needs of a diverse workforce as well as to maximize the potential of all workers, so that San Francisco can be well positioned for the demands of the 21 st century?

Most people believe in the golden rule: treat others as you want to be treated. The implicit assumption is that how you want to be treated is how others want to be treated. But when you look at this proverb through a diversity perspective, you begin to ask the question: what does respect look like; does it look the same for everyone? Does it mean saying hello in the morning, or leaving someone alone, or making eye contact when you speak?

It depends on the individual. We may share similar values, such as respect or need for recognition, but how we show those values through behavior may be different for different groups or individuals. How do we know what different groups or individuals need? Perhaps instead of using the golden rule, we could use the platinum rule which states: "treat others as they want to be treated." Moving our frame of reference from what may be our default view ("our way is the best way") to a diversity-sensitive perspective ("let's take the best of a variety of ways") will help us to manage more effectively in a diverse work environment.

\section{An Organization Role}

You have a key role in transforming the organizational culture so that it more closely reflects the values of our diverse workforce. Some of the skills needed are:

- $\quad$ an understanding and acceptance of managing diversity concepts

- $\quad$ recognition that diversity is threaded through every aspect of management

- Self-awareness, in terms of understanding your own culture, identity, biases, prejudices, and stereotypes

- willingness to challenge and change institutional practices that present barriers to different groups

It's natural to want a cookbook approach to diversity issues so that one knows exactly what to do. Unfortunately, given the many dimensions of diversity, there is no easy recipe to follow. Advice and strategies given for one situation may not work given the same situation in another context.

Managing diversity means acknowledging people's differences and recognizing these differences as valuable; it enhances good management practices by preventing

Managing Diversity is Different from Affirmative Action 
Managing diversity focuses on maximizing the ability of all employees to contribute to organizational goals. Affirmative action focuses on specific groups because of historical discrimination, such as people of color and women. Affirmative action emphasizes legal necessity and social responsibility; managing diversity emphasizes business necessity. In short, while managing diversity is also concerned with underrepresentation of women and people of color in the workforce, it is much more inclusive and acknowledges that diversity must work for everyone.

\section{Contribution Of Diverse Workforce Towards Organization}

1) As a Social Responsibility

Because many of the beneficiaries of good diversity practices are from groups of people that are "disadvantaged" in our communities, there is certainly good reason to consider workforce diversity as an exercise in good corporate responsibility. By diversifying our workforces, we can give individuals the "break" they need to earn a living and achieve their dreams.

2) As an Economic Payback

Many groups of people who have been excluded from workplaces are consequently reliant on tax-supported social service programs. Diversifying the workforce, particularly through initiatives like welfare-to-work, can effectively turn tax users into tax payers. So in this way it prove to be an economic payback and support the organization.

3) As a Resource Imperative

The changing demographics in the workforce, that were heralded a decade ago, are now upon us. Today's labor pool is dramatically different than in the past. No longer dominated by a homogenous group of white males, available talent is now overwhelmingly represented by people from a vast array of backgrounds and life experiences. Competitive companies cannot allow discriminatory preferences and practices to impede them from attracting the best available talent within that pool.

4) As a Legal Requirement

Many companies are under legislative mandates to be non-discriminatory in their employment practices. Non-compliance with Equal Employment Opportunity or Affirmative Action legislation can result in fines and/or loss of contracts with government agencies. In the context of such legislation, it makes good business sense to utilize a diverse workforce.

5) As a Marketing Strategy

Buying power, particularly in today's global economy, is represented by people from all walks of life (ethnicities, races, ages, abilities, genders, sexual orientations, etc.) To ensure that their products and services are designed to appeal to this diverse customer base, "smart" companies, are hiring people, from those walks of life - for their specialized insights and knowledge. Similarly, companies who interact directly with the public are finding increasingly important to have the makeup of their workforces reflect the makeup of their customer base.

6) As a Business Communications Strategy

All companies are seeing a growing diversity in the workforces around them - their vendors, partners and customers. Companies that choose to retain homogenous workforces will likely find themselves increasingly ineffective in their external interactions and communications. And an effective communication system developed in the organization which help the concern to over come the communication barriers.

7) As a Capacity-building Strategy

Tumultuous change is the norm in the business climate of the 21 stcentury. Companies that prosper have the capacity to effectively solve problems, rapidly adapt to new situations, readily identify new opportunities and quickly capitalize on them. This capacity can be measured by the range of talent, experience, knowledge, insight, and imagination available in their workforces. In recruiting employees, successful companies recognize conformity to the status quo as a distinct disadvantage. In addition to their job-specific abilities, employees are increasingly valued for the unique qualities and perspectives that they can also bring to the table. According to Dr. Santiago Rodriguez, Director of Diversity for Microsoft, true diversity is exemplified by companies that "hire people who are different - knowing and valuing that they will change the way you do business."

For whichever of these reasons that motivates them, it is clear that companies that diversify their workforces will have a distinct competitive advantage over those that don't. Further, it is clear that the greatest benefits of workforce diversity will be experienced, not by the companies that that have learned to employ people in spite of their differences, but by the companies that have learned to employ people because of them.

\section{Reasons For Diversity Management}

1) Talent Shortage: Diversity management can reduce unwelcomed turn over and reduce absenteeism. In order to get the best out of the workforce,companies should not exclude any particular group by gender, race, or 
religion, the company will be excluding the person that is going to produce the next famous and successful product or someone who is going to contribute in another useful way in marketing, computing or engineering or elsewhere.

2) Range of Consumer Need: In recent years, organisations have recognised that they can best serve different customer groups in many different markets in more diverse workforce even if the locality or home base of the organisation is located in a culturally imaginous market, there may be substantial customers that are located in other more diverse communities either in its home country or abroad. In communities where other languages dominate, organisations benefits from hiring employees who speak the dominant languages. They could also have diverse workforces design products that attract diverse consumers or customers.

3) Globalisation: Globalisation can be defined as a process where organisations or businesses start to operate in an international scale. Companies are more open to internationalise their operations, they choose an efficient location to operate. Prices of inputs vary all over the world, which attracts companies to shift some of their production to countries with cheaper inputs such as labour in developing countries but are usually limited to save countries. Workforce diversity is a critical measure to companies who seeks to establish themselves. (Globalisation and business 2013)

\section{Consequences Of Ignoring Diversity}

Ignoring diversity issues costs time, money, and efficiency. Some of the consequences can include unhealthy tensions; loss of productivity because of increased conflict; inability to attract and retain talented people of all kinds; complaints and legal actions; and inability to retain valuable employees, resulting in lost investments in recruitment and training.discrimination and promoting inclusiveness. Good management alone will not necessarily help you work effectively with a diverse workforce. It is often difficult to see what part diversity plays in a specific area of management.The Office of Affirmative Action, Equal Opportunity and Diversity is experienced in providing help with training and advice on the variety of situations that occur, tailored to your specific environment.To illustrate, the following two examples show how diversity is an integral part of management. The first example focuses on the area of selection, the second example looks at communication:

\section{Issues Which Need To Be Focused}

- How do you make the job sound appealing to different types of workers?

- How can recruitment be effectively targeted to diverse groups?

- How do you overcome bias in the interviewing process, questions, and your response? Strategies adopt to manage diverse work force

- Specify the need for skills to work effectively in a diverse environment in the job, for example: "demonstrated ability to work effectively in a diverse work environment."

- Make sure that good faith efforts are made to recruit a diverse applicant pool.

- Focus on the job requirements in the interview, and assess experience but also consider transferable skills and demonstrated competencies, such as analytical, organizational, communication, coordination. Prior experience has not necessarily mean effectiveness or success on the job.

- Use a panel interview format. Ensure that the committee is diverse, unit affiliation, job classification, length of service, variety of life experiences, etc. to represent different perspectives and to eliminate bias from the selection process. Run questions and process by them to ensure there is no unintentional bias.

- Ensure that appropriate accommodations are made for disabled applicants.

- Know your own biases. What stereotypes do you have of people from different groups and how well they may perform on the job? What communication styles do you prefer? Sometimes what we consider to be appropriate or desirable qualities in a candidate may reflect more about our personal preferences than about the skills needed to perform the job.

\section{'Fair Treatment' is not equal to 'same treatment'}

Many people think that "fairness" means "treating everyone the same." How well does treating everyone the same work for a diverse staff? For example, when employees have limited English language skills or reading proficiency, even though that limit might not affect their ability to do their jobs, transmitting important information through complicated memos might not be an effective way of communicating with them. While distributing such memos to all staff is "treating everyone the same," this approach may not communicate essential information to everyone. A staff member who missed out on essential information might feel that the communication process was "unfair." A process that takes account of the diverse levels of English language and 
reading proficiency among the staff might include taking extra time to be sure that information in an important memorandum is understood. Such efforts on the part of supervisors and managers should be supported and rewarded as good management practices for working with a diverse staff.

\section{Barriers For Accepting Work Force Diversity}

1) Prejudice: an unfair and unreasonable opinion or feeling, especially when formed without enough thought or knowledge; an unjustified negative attitude toward a person based on his or her membership in a particular group

2) Ethnocentrism: a tendency to regard one's own group, culture, or nation as superior to others this tendency of the work force prove a big barrier in accepting a policy of diverse work force.

3) Stereotypes: a fixed idea about what a particular type of person is like, especially an idea that is wrong; a set of beliefs about a group that is applied universally to all members of that group

4) Blaming the victim: making incorrect casual attributions linking aggressions with perceived characteristics of groups

5) Discrimination: barring an individual from membership in an organization or from a job because of his or her membership of a particular group. Discriminating behavior of work force restrict them to adopt new environment.

6) Harassment: consciously shunning verbally or physically abusing an individual because of membership in a particular group

7) Backlash: negative reaction to members of previously underrepresented groups gaining power and influence

1. By reducing prejudices and use of stereotypes

\section{Strategies To Increae Awareness About Work Force Diversity}

- Recognizing that diversity exists and learning to value and respect fundamental differences

- $\quad$ Admitting to one's own biases and prejudices and committing to reduce them

- Dismissing myths about diverse others when in a group of friends or associates

2. By minimizing miscommunication with diverse others

- $\quad$ Educating oneself about differences by reading, listening, and broadening one's experience base about diverse people

- $\quad$ Communicating effectively by listening attentively and asking questions about what one did not understand

- $\quad$ Avoiding terms that spotlight certain groups and imply the individual is an exception

- Avoiding valuing one's message based on dress, mannerisms, accent, or eye contact

3. By building relationships with diverse others

- $\quad$ Forming positive relationship with diverse others

- $\quad$ Seeking feedback from diverse others about how well one is communicating respect for them and valuing their diversity

- Treating diverse others as invited guests by showing interest in them rather than treating them as strangers

\section{Conclusion}

Workforce diversity represents both a challenge and an opportunity for business. A growing number of progressive organisations are realizing the need for valuing diversity in the workforce, so as to ensure strategic utilization of human resources for the accomplishment of strategic goals. The extent to which managers recognize diversity and its potential advantages and disadvantages defines an organization's approach to managing the diversity. No organisation in this world of globalization would survive without workforce diversity. It is believed that organizations should put in place strategies to enhance workforce diversity. In terms of organizational learning, organizations are still stuck on the problem of getting people to value diversity and have not yet determine the ways to utilize and exploit it. It is the approach to diversity, not the diversity itself which determines the actual positive and negative outcomes. Finally, creating a diverse workforce takes time and even longer to reap the benefits. The management and leaders must not lose focus and interest in creating a diverse workforce due to the lack of immediate returns. 


\section{References}

[1]. Al-Lamki S. M.(2002). Orientation: The essential ingredient in cross-cultural management, International Journal of Management, 19, 568

[2]. Cox, Taylor, Jr. Cultural Diversity in Organizations. Berrett-Koehler Publishers, 1993

[3]. Dike, Priscilla (2013) The Impact of Workforce Diversity on organization

[4]. http://ucsfhr.ucsf.edu

[5]. Patrick H.Andrew , Kumar V. Raj (2012). Managing Workplace Diversity. Sage journal

[6]. Rob McInnes, Diversity World 1999 (Workforce Diversity: Changing the Way You Do Business)

[7]. Seyed-Mahmoud Aghazadeh, (2004) "Managing workforce diversity as an essential resource for improving organizational performance", International Journal of Productivity and Performance Management, Vol. 53 Iss: 6, pp.521 - 531

[8]. www.aaeod.ucsf.edu.

[9]. Www.ask.com

[10]. www.ehow.com

[11]. www.tamu.edu 